\section{SATISFYING ORTHODONTIC DEMAND}

If you want to meet patient demand for affordable cosmetic orthodontic treatment, would like to benefit from receiving your whole fee before treatment commences and have the reassurance of specialist support, look no further than Oralign Ltd.

Oralign is unlike other orthodontic treatment undertaken by GDPs, in that Oralign's specialist orthodontists undertake the diagnosis, complete the treatment planning and recommend which of Oralign's three appliances is best suited for the case. All of this gives GDPs the reassurance they need and safeguards against unexpected tooth movement and unforeseen difficulties.

Via this unique online diagnostic, treatment planning and case support service, general practitioners are able to treat non-extraction, cosmetic cases, confident in the fact that they will be fully supported throughout treatment by one of the registered orthodontic specialists at Oralign Ltd.
Oralign Ltd not only provides clinical flexibility so you can treat the majority of limited treatment orthodontic cases in your practice, but also offers an interest free, patient funding option through financial partners Chrysalis Dental Finance.

So whilst your patients can afford the smile they've always dreamed of, you will feel the full benefit of improved cash flow thanks to an up-front fee, with no lab fees or additional materials costs to worry about.

Oralign Ltd will be running GDP courses in the Oralign technique throughout 2014. For more information visit the website at www.oralign.co.uk.

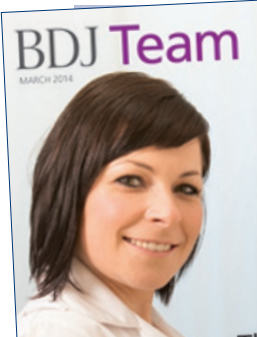

in clinical dentistry

BDA radiographs

\section{TEN FREE HOURS OF CPD FOR DENTAL PROFESSIONALS}

At the end Team is aimed at the whole dental team and will be of March published every month on a newly-launched website, the $B D J$ launched a brand new sister publication, $B D J$ Team. BDJ Team

will offer dental care professionals (DCPs) ten free hours of continuing professional development (CPD) in 2014.

The successor to Vital, BDJ published every month on a newly-launched website, December 2014 a set of multiple choice questions based on an article published in BDJ Team will be available at www.nature.com/bdjteamcpd, enabling users to log on, answer the questions then download or print off their verifiable CPD certificate.

The first CPD article in BDJ Team was on the core topic of radiography, and this will be followed closely by a second core topic, infection control, with an article on clinical waste management.

To find out more about BDJ Team, visit www. nature.com/bdjteam. To request free access to $B D J$ Team CPD, visit www.nature.com/bdjteamcpd.

\section{SUPPORTING WORLD ORAL HEALTH DAY}

Listerine was proud to support the 2014 World Oral Health Day on 20 March. The theme of this year's event was 'celebrating healthy smiles', to raise awareness of the importance of establishing and maintaining a proper oral care routine. The makers of Listerine offered tips for establishing healthy habits for a lifetime, which include taking a few minutes each day to brush, floss and rinse with a therapeutic mouthwash like Listerine.

The Listerine Advanced Defence mouthwashes from Johnson \& Johnson are scientifically proven adjuncts to professional treatment, designed to help treat and prevent specific oral care conditions. The range includes Advanced Defence Sensitive, Advanced Deference

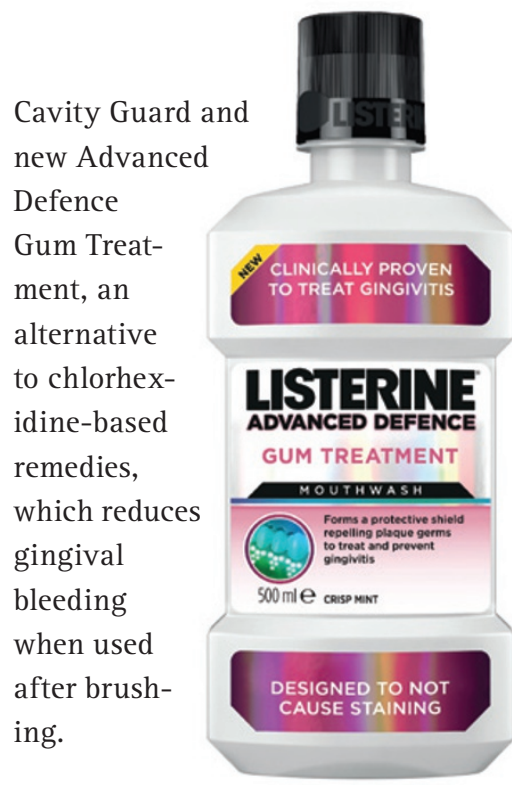

\section{NEW COURSES FOR THE WHOLE TEAM}

To help the whole dental team keep up to date Henry Schein Dental is pleased to announce their new and extensive Education and Development programme. Courses include:

Infection Control - A must for the whole dental team, this course is specifically designed to meet HTM 01-05 and CQC audit requirements and provides a clear understanding of why the implementation of effective cross-infection control procedures are so important.

Laser Technology - Four intensive courses for differing levels of experience: 'Introduction to Lasers in Dentistry'; 'Diode Laser in Dentistry'; 'Periodontal Laser Surgery'; and 'Lasers and CEREC'.

Contemporary Endodontics Introducing two new courses: 'New to Rotary' is a beginners' course aimed at clinicians who are new to rotary/reciprocation. 'Contemporary Endodontics' is an advanced course aimed at clinicians confident in endodontics looking to take their skills to a higher level.

Whitening - Four evening courses covering a spectrum of topics: 'Complex Bleaching Cases'; 'Getting Your Patients to Say 'Yes' to Bleaching'; '7 Habits of Highly Effective Whitening Practice' and 'Implement a Team Approach to Bleaching'.

Courses run throughout the year at convenient locations nationwide and offer exceptional value for money and the opportunity to gain valuable verifiable CPD. Other courses on hot topics are added at any time, so for the latest schedule and full course details visit www.henryschein.co.uk, call Sue 0'Rourke on 0292044 2818 or email suzanne.orourke@henryschein.co.uk. 